\title{
Prácticas de campo planificadas en la formación inicial de futuros profesores de biología y su aporte a la construcción del conocimiento profesional: el caso de primer semestre
}

Field works designed in the initial formation of future biology teachers and its contribution to the construction of the professional knowledge: the case of first semester

\section{Elías Amórtegui, Mónica Correa y Édgar Valbuena}

${ }^{1}$ Estudiante de décimo semestre de Licenciatura en Biología. Universidad Pedagógica Nacional. ${ }^{2}$ Profesor del Departamento de Biología. Universidad Pedagógica Nacional. Grupo de Investigación Conocimiento Profesional del Profesor de Ciencias. folkerpan@hotmail.com; macs1986@hotmail.com; edgarorlay@hotmail.com

\section{Resumen}

Presentamos resultados parciales de la investigación Las Prácticas de Campo planificadas en el Proyecto Curnicular de Licenciatura en Biología de la Universidad Pedagógica Nacional, caracterización desde la perspectiva del Conocimiento Profesional del Profesor de Biología. Dicha investigación pretende caracterizar las Prácticas de Campo planificadas por parte de los formadores de formadores y su aporte a la construcción del Conocimiento Profesional de los futuros profesores de Biología. Para este caso, concretamente nos referimos a la planificación de las Prácticas de Campo de primer semestre con base en las guías, el documento de presentación del semestre y los programas de los espacios académicos del semestre involuarados en la salida de campo. La caracterización se llevó a cabo desde un enfoque cualitativo, a partir del método de análisis de contenido, teniendo en cuenta un sistema de categorías empleado para el análisis de Trabajos Prácticos en la enseñanza de la Biología. Encontramos resultados con respecto a la tipología del trabajo, la integración de espacios académicos, los referentes epistemológicos y la reflexión docente en la formación inicial.

Palabras clave

Trabajo Práctico (TP), Prácticas de Campo (PC), Conocimiento Profesional del Profesor de Biología (CPPB), Enseñanza de la Biología, Formación inicial de profesores, Proyecto Curricular de Licenciatura en Biología (PCLB).

\section{Abstract}

We present partial results of the work called Las Prácticas de Campo planificadas en el Proyecto Curniaular de Licenciatura en Biología de la Universidad Pedagógica Nacional, caracterización desde la perspectiva del Conocimiento Profesional del Profesor de Biología. This investigation pretends to describe the field Works designed by teachers and its contributions to the construction of the Professional Knowledge of the future Biology teachers. In this case we refer to the case of first semester basing in field work's guides, documents for the semester, and programs of academic spaces. The description is made in a qualitative focus, by the content analysis method, with a categories system for the analysis of practice works in Biology teaching. We found results about the typology of the work, the academic spaces integration, the epistemologic thoughts and the teacher reflection.

Key words

Practice Work, Field Work, Proffessional Knowledge of the Biology Teacher, Biology Teaching, Teacher's initial formation, Curriculum Project of the Biology Degree. 


\section{Introducción}

Se ha subestimado socialmente la labor de los maestros al considerarse que para enseñar sólo es necesario tener dominio del conocimiento disciplinar a profundidad, dejando de lado el hecho de que nosotros como profesionales de la educación, poseemos un conocimiento particular que además de induir lo disciplinar, induye elementos pedagógicos, didácticos, contextuales, epistemológicos, entre otros, lo cual nos posibilita el enseñar adecuadamente, diferente a como lo pueden realizar los profesionales de otras áreas (Valbuena, 2007).

Precisamente considerando que los problemas referentes al estatus de la profesión docente están relacionados con el poco desarrollo en la producción del Conocimiento Profesional del Profesor, actualmente se están realizando diversas investigaciones que pretenden aportar a la solución de dicha problemática. Como resultado de las mismas, se plantea la importancia de investigar en el ámbito de la formación docente los procesos que promueven la construcción de dicho conocimiento y su vinculación en la formación inicial de maestros (Tardif, 2004). Considerando la formación inicial como la base sobre la cual se empieza a generar el conocimiento profesional, es relevante investigar las actividades, evaluaciones, planes de trabajo, que se plantean en los programas curriculares de formación de profesores, siendo aún mas importante trabajarlo en el contexto de una institución ayya principal misión es la formación de docentes, como lo es la Universidad Pedagógica Nacional. Así en esta ponencia hacemos referencia a la formación inicial de profesores de Biología en el marco del PCLB.

En cuanto al Trabajo Práctico y la formación del profesorado en la enseñanza de las Ciencias, éste ha venido constituyéndose en una faceta muy valorada entre los enseñantes (Boyer y Tiberghien 1989; Rodrigo 1993; Garća Barros 1998; citados en M. Rodrigo et al 1999); no obstante los estudios realizados sobre la incidencia de estas actividades, son escasos (Rodrigo et al, 1999). Cabe señalar que existen diversas dasificaciones de los Trabajos Prácticos en la Enseñanza de las Ciencias, predominando: las Prácticas de Laboratorio, las Prácticas de Campo y los Ejercicios de Lápiz y Papel. Ahora bien, las Prácticas de Campo son una faceta muy valorada en la formación inicial de profesores de Ciencias (Rodrigo et al, 1999), al concebirse como una estrategia trascendental en la enseñanza de las mismas y por ende de la Biología, que permiten relacionar la teoría con la práctica y favorecer el aprendizaje de diversos contenidos, constituyendo además una fuente de: información directa, ejemplos y experiencias contextualizadas (Del Carmen y Pedrinad 1997). Es importante analizar el papel de las Prácticas de Campo en los programas curriculares de formación de docentes en Ciencias; sin embargo es de destacar que existen pocas investigaciones sobre la incidencia de dichas actividades y mucho menos sobre su aporte a la construcción del conocimiento de los profesores. De los pocos estudios se abarca en mayor proporción las prácticas de laboratorio.

En el PCLB se realizan Prácticas de Campo para cada semestre, considerándose importantes en la integración de sus espacios académicos; sin embargo no se han realizado trabajos que permitan su caracterización, análisis y sistematización, de tal manera que se de cuenta de las inquietudes que existen sobre su pertinencia, su aporte a la formación docente, a la integración de los espacios académicos de los diversos semestres, su secuencia, entre otros. Es importante resaltar la incidencia de los procesos formativos en el cambio de referentes de los profesores acerca del Conocimiento Profesional del Profesor, dado que muchas veces los docentes reproducen ó eliminan en su práctica las maneras de enseñar que experimentaron cuando fueron estudiantes, puesto que a partir de dicha experiencia construyen diversos referentes epistemológicos acerca de la enseñanza, del aprendizaje, de las metodologías y actividades de enseñanza, de la evaluación de los aprendizajes, entre otros (Grossmann, 1990). 
De acuerdo con lo anterior, se realizó la investigación con el objetivo de caracterizar las Prácticas de Campo planificadas en el Proyecto Curricular de Licenciatura en Biología de la Universidad Pedagógica Nacional durante el periodo 2008-II y su aporte a la construcción del CPPB. De este modo en el presente artículo por cuestiones de espacio, se presenta exdusivamente la caracterización de primer semestre.

\section{Desarrollo}

Nuestra investigación se identificó con una perspectiva cualitativa. Para el proceso de sistematización, empleamos el método del análisis de contenido a partir de la información de las guías de campo y los programas de los espacios académicos elaborados por los formadores, y de una entrevista semiestructurada realizada a la coordinadora del semestre. Desarrollamos el trabajo en tres fases: selección de las categorías para el análisis de contenido y contextualización; obtención de datos; y sistematización de datos y análisis de resultados. Para el análisis de la información, seleccionamos el sistema de categorías diseñado por Puentes (2008), induyendo además unas categorías emergentes como resultado de nuestra investigación (Ver tabla 1).

\begin{tabular}{|c|c|}
\hline Categoría & Subcategorías \\
\hline \multirow[t]{3}{*}{ 1. NATURALEZA } & 1.1 Imagen de práctica \\
\hline & 1.2 Relación teoría- práctica \\
\hline & 1.3 Clase de Trabajo Práctico \\
\hline \multicolumn{2}{|l|}{ 2. FINALIDADES } \\
\hline \multirow[t]{4}{*}{ 3. METODOLOGÍA } & 3.1 Estructura de la Guía de Campo \\
\hline & 3.2 Enfoque \\
\hline & 3.3 Momento de realización \\
\hline & 3.4 Roles \\
\hline \multirow[t]{3}{*}{ 4. EVALUACIÓN } & 4.1. Tipos \\
\hline & 4.2 Roles \\
\hline & 4.3 Contenidos a evaluar \\
\hline \multirow{4}{*}{$\begin{array}{l}\text { 5. RELACIÓN CON ASPECTOS } \\
\text { EPISTEMOLÓGICOS }\end{array}$} & 5.1 Biología como Ciencia \\
\hline & 5.2 Objeto de estudio de la Biología \\
\hline & 5.3 Producción de conocimiento biológico \\
\hline & 5.4 Imagen de Ciencia \\
\hline \multicolumn{2}{|l|}{$\begin{array}{l}\text { 6. ASPECTOS RELACIONADOS CON LA } \\
\text { REFLEXIÓN DOCENTE }\end{array}$} \\
\hline $\begin{array}{l}\text { 7. ASPECTOS RELACIONADOS CON EL } \\
\text { CURRÍCULO DEL PCLB }\end{array}$ & \\
\hline
\end{tabular}

Tabla 1. Sistema de categorías para el análisis de los Trabajos Prácticos en la enseñanza de la Biología. Modificado de Puentes (2008) (En negrilla las categonías incorporadas como resultado de nuestra investigación).

Realizamos la sistematización de los datos obtenidos teniendo en cuenta el método de análisis de contenido. En primera instancia extrajimos unidades de información de cada una de las fuentes, posteriormente las agrupamos en el sistema de categorías y finalmente formulamos las proposiciones y referentes. A continuación presentamos los resultados hallados, describiendo para cada una de las categorías los referentes de los formadores', un ejemplo y su análisis, particularmente para el caso de primer semestre.

- Entiéndase formador como el docente que enseña a los futuros profesores de Biología.

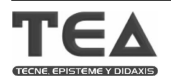




\section{Naturaleza del trabajo práctico}

Imagen de Práctica: Los formadores establecen la práctica como un espacio académico, riguroso, de vivencia fuera del aula que posibilita interactuar con organismos y pares académicos e interrelacionar, comprender y encontrar evidencias de lo teórico con respecto tanto a lo biológico como a lo pedagógico.

"[definiendo la salida de campo] (...) es un espacio académico riguroso y obligatorio en donde los estudiantes tienen la oportunidad de trasladarse a otros escenarios en donde podrán interactuar con otros organismos y pares académicos en pro de profundizar y consolidar sus conocimientos y prácticas del entomo educativo (....)"

Desde la perspectiva de la formación de profesores, lo práctico responde a la integración de diferentes componentes, lo académico, la experiencia y la cosmovisión, en ese sentido la práctica no se limita exdusivamente a un compilado de técnicas, sino que lo desborda e induye una gama de aspectos como diversos conocimientos (Garća, 2006).

Relación Teoría-Práctica (T-P): Los formadores consideran que debe existir una relación explíata e integral entre teoría y práctica, considerando en algunos casos la teoría como un referente de análisis para la práctica y ésta como un elemento de contrastación y validación de la teoría.

"[haciendo referencia a la Práctica de campo] (...) desarrollar un trabajo académico $y$ práctico (...) que es visto de manera explicita, integral y transdisoiplinar en un proyecto de semestre y en el reconocimiento de la realidad a través de la salida de campo. "

Este referente se corresponde con un enfoque constructivista, en el que se establece una relación integral entre T-P, de manera tal que se trasciende del modelo de transmisión-recepción en donde dicha relación se presenta fundamentalmente en una sola vía, por lo que la práctica se constituye como complemento de la enseñanza teórica que es transmitida por el docente. De este modo, es importante promover una visión donde las prácticas no corresponden a la simple explicación y confirmación de la teoría, más bien se debe considerar que las relaciones teoríapráctica, deben guardar proporciones con el trabajo que se realiza en la actividad cotidiana, donde las actividades prácticas se desarrollan como respuesta a preguntas y/o problemas y para poder realizarlas primero es indispensable proveer referentes teóricos que den sentido a las actividades mismas (Puentes, 2008).

Uno de los aspectos que genera mayor controversia en la formación docente, está relacionado con las relaciones entre la teoría y la práctica, en general ambos elementos han sido considerados como dimensiones separadas pero siempre atribuyéndole superioridad a la teoría sobre la práctica. De este modo, es importante que en la formación docente se reflexione sobre la forma en que se aborda la teoría y la práctica, partiendo de la idea de tratarlas como campos mutuamente constituyentes y relacionados. De esta manera la relación entre teoría y práctica no supone que la teoría implique la práctica, se derive de ella o la refleje, sino que, la teoría transforma la práctica y esta a su vez puede transformar la teoría (Moreno et al, 2006).

Clase de Trabajo Práctico: En el desarrollo de la guía de campo, los formadores induyen en una mayor proporción, ejercicios cenrados planteados exdusivamente por ellos, en los que se solicita a los futuros licenciados, describir (fósiles, tiempo geológico, ecosistemas, organismos), definir (ecosistemas, lixiviación), analizar, inferir (procesos de fosilización, caracteństicas de

\footnotetext{
${ }^{1}$ El texto resaltado en cursiva y entre comillas, corresponde a afirmaciones tomadas textualmente de los documentos revisados. 
lugares del pasado, procesos de extinción), relacionar conceptos (tiempo geológico con fosilización, aultura con naturaleza y cosmovisión), diligenciar tablas y registrar datos (relacionados con características de plantas y animales, características ambientales y concepciones sobre educación y Ciencias naturales).

"[Elemento de la guía anexa relacionada con la visita al Museo Acuña] Describe los fósiles observados y a partir de ello infiere su proceso de fosilización y relaciónalo con el tiempo geológico"

Según Puentes (2008), este referente da cuenta de un tipo de TP basado en ejercicios, en donde se utilizan problemas de carácter cenado, los cuales están planteados siempre por el docente y los estudiantes deben seguir protocolos también determinados por el profesor para hallar una solución que él previamente conoce y que puede considerar como la única respuesta. Ante esto, los estudiantes deben realizar consultas bibliográficas que corroboren los resultados obtenidos o que confirmen lo que el profesor expone frente a los resultados que obtienen los estudiantes. En este tipo de práctica, los estudiantes logran desarrollar habilidades motrices y de seguimiento de instrucciones a través de la realización estricta de protocolos proporcionados, en los cuales se estipulan daramente los materiales y método a emplear. A partir de esta clase de prácticas, el futuro licenciado puede comprobar algunos elementos teóricos, pero sin hacer una abstracción real de lo que ocurre o sin que tenga real significado, ya que como tal la experiencia no parte de una situación que resulte problemática para él.

Teniendo en cuenta que en el Modelo del profesor investigador, con el que se identifica la presente investigación, es deseable aportar por la formación de un profesor reflexivo, con capacidades en la investigación, que implica asumir situaciones problemáticas ante las cuales el profesor debe elaborar criterios racionales de comprensión y propuestas argumentadas que se materialicen en proyectos de intervención (García, 2006), de acuerdo con Puentes (2008) se considera que el tipo de prácticas relacionadas con la investigación, serían el ideal de trabajo con los futuros docentes.

\section{Finalidades del trabajo práctico}

Los formadores plantean e identifican como finalidades de las Prácticas de Campo, propósitos conceptuales, procedimentales y actitudinales relacionadas tanto con la formación disciplinar Biológica, cultural y social, como con la formación docente.

"[Objetivo de la Práctica de Campo] (...) Reflexionar sobre los diferentes espacios de vida que se encontrarán durante su recornido y cómo estos hacen parte de su proceso formativo como ciudadanos del mundo y futuros educadores (...)"

Desde la perspectiva de Gil y Valdés (1996), dicho referente se acerca a un tipo de finalidad de Resolver problemas, dado que no se centra exdusivamente en la realización de los procedimientos experimentales, sino además, induye aspectos de la actividad cientúfica, y permite a los estudiantes tomar decisiones que les permitan transformar y solucionar problemas dentro de un contexto espećfico, en este caso es de resaltar que dichos problemas se encuentran enmarcados en la formación de docentes de Biología. Según Puentes (2008), este tipo de finalidades le permitan al estudiante a partir de su conocimiento cotidiano y de sus intereses y/o necesidades proponer y solucionar situaciones problémicas.

\section{Metodología}

Estructura de la guía de campo: Los formadores incluyen en sus guías de campo aspectos como: elementos contextuales, objetivos de aprendizaje, temáticas de trabajo, cronograma de 
actividades, materiales de trabajo, elementos teóricos, recomendaciones, métodos y herramientas, costos, bibliografía y guías anexas.

"[Haciendo referencia las recomendaciones para la PC] Agenda de Campo, Brújula, Lápiz, Botiquín, Guías de trabajo, Mapas de ubicación por grupo y Tijeras..."

La guía de campo es sólo un instrumento de trabajo que debe orientar en forma clara la actividad individual y grupal de los estudiantes, por lo tanto es vital que su diseño responda a un plan ordenado de labores escolares. Esto no debe reemplazar al maestro, por el contrario, debe conformar un plan conjunto, que lo involucre como orientador en la búsqueda de conocimientos, adquisición de habilidades y destrezas. Sin embargo es necesario que induyan todos los aspectos necesarios para una buena Práctica de Campo, la ubicación espacio-temporal, los objetivos, los materiales y equipos, procedimientos, indicadores de evaluación, textos de consulta, anexos, entre otros (Alarcón y Piñeros, 1989).

Enfoque: Los formadores dan cuenta de diferentes enfoques en las Prácticas de Campo, sin embargo en su mayoría, acerca de un enfoque expositivo, en el que se plantean procesos algorítmicos con ejercicios cerrados en los que se induyen actividades como describir, definir, observar, analizar, reflexionar, inferir, relacionar, registrar datos y diligenciar tablas.

"[Haciendo referencia a las salidas de campo como estrategia metodológica del componente] Éstas tienen como objetivo, ir adentrando a los estudiantes al trabajo en campo de un biólogo (...) mirar, ver y observar, (...) plantear preguntas (...) una actítud auto reflexiva $y$ conciente de las formas en que se construye el conocimiento en las Ciencias (...)"

Este enfoque expositivo no se permite la participación del estudiante en el diseño y preparación de la actividad. Aquí los estudiantes comprueban o corroboran aquellos datos que han sido expuestos por su profesor; a partir de lo que plantea el profesor, los estudiantes desarrollan algunas habilidades en el manejo y/o manipulación de materiales y técnicas de campo 0 de laboratorio (Puentes, 2008). Se debe tener en auenta que dado que es el primer semestre de la formación inicial, no todos los futuros profesores han realizado trabajos prácticos en su educación secundaria, por lo que el trabajo del formador debe estar orientado de una forma mas expositiva que desde un enfoque investigativo. En concordancia con la subcategońa Clase de Trabajo Práctico, se considera que los enfoques relacionados con la investigación, serían el ideal de trabajo con los futuros docentes.

Momento de realización: Los formadores consideran que la Práctica de Campo debe realizarse después de haber abordado lo teórico, pues éste es un referente para analizar la práctica.

"[Con respecto al momento de realización de la salida] (...) pensar la salida de campo en un determinado tiempo y eso indica que se hayan abarcado esas temáticas, por eso casi siempre la colocamos dos meses después de haber abordado las temáticas porque si vamos a la Práctica de Campo sin ese conocimiento conceptual de lo epistemológico, de las Ciencias, de saber cientrfico (...)"

Este referente concuerda de nuevo con un Enfoque Expositivo, en donde se puede considerar que no se realizarán Trabajos Prácticos al inicio de una temática, puesto que se concibe que el estudiante debe tener unos conocimientos científicos daros antes de realizar dichos trabajos (Puentes, 2008). Para el caso de primer semestre se tienen en cuenta lo social y cultural.

Roles: Los formadores plantean con respecto a los roles asumidos por los actores de la Práctica de Campo una relación Instructor-Reproductor, en donde el futuro licenciado ejecuta la guía de 
campo, observa, toma nota, realiza las actividades planteadas y al final retroalimenta la guía de campo, mientras que el formador plantea la guía de campo, proporciona conocimientos al estudiante y orienta el trabajo.

"En la práctica, el estudiante ejecula absolutamente todo, es decir que la orientación de los maestros es simplemente orientarlos en la indicación, en la ejecución de, pero ellos son los que con su observación y con la importancia que le den a la salida logran ejecutar la guía de campo (...)"

De acuerdo a Rodrigo et al(1999), este tipo de roles del estudiante y del docente corresponden, como se ha visto en las categorías de Enfoque y Finalidades, a un tipo de trabajo Expositivo, en el que el docente fundamentalmente planea, dirige y propone las actividades, mientras que el estudiante las realiza, sigue protocolos, en donde asimila y reproduce el conocimiento.

Considerando que los futuros licenciados deben diseñar en su futura labor docente Prácticas de Campo, al no permitirse la participación del futuro docente en el diseño y preparación de la guía de campo, es posible que no se propicie que el futuro docente comprenda las Prácticas de Campo como una estrategia didáctica para la enseñanza de la Biología, además no se le está brindando la posibilidad de poner en práctica los conocimientos pedagógicos, didácticos, biológicos que ha ido adquiriendo durante su formación.

Es de resaltar que desde los enfoques relacionados con la investigación, y participando en la planeación de las metodologías de las Prácticas de Campo, el futuro licenciado es capaz de identificar o reconocer las limitaciones de su conocimiento, de manera que reconoce que es necesario replantear dicho conocimiento y asume nuevas posiciones de acuerdo con lo que va construyendo bajo la guía del formador (Puentes, 2008).

\section{Evaluación}

Tipos: Los formadores de primer semestre dan cuenta de un tipo de evaluación formativa en la que se valora el trabajo del futuro licenciado durante el desarrollo de la actividad involucrando el antes, durante y después de la Práctica de Campo.

"(..) La evaluación se reconoce como el seguimiento constante del proceso de los estudiantes durante $\theta$ semestre, éste, aunque finalmente es avantificado, no deja de ser un proceso de seguimiento cualitativo que se realiza no sólo por parte de la maestra, sino que además, cuenta con la participación del grupo y de cada estudiante, en lo que se denomina coevaluación(...)"

Este referente va más allá de un tipo de evaluación inicial en la que se busca establecer si el estudiante posee los conocimientos básicos que se necesitan para desarrollar la actividad; por el contrario corresponde a un tipo de evaluación formativa, en la que se valora el trabajo del estudiante durante el desarrollo de la actividad (Puentes, 2008 y Geli, 1995); este tipo de evaluación se debe considerar fundamental en la formación de profesores de Biología, pues permite realizar procesos de autorreflexión.

Roles: Los formadores de formadores consideran como roles en la evaluación tanto al formador como al futuro profesor el grupo en el que se encuentra (autoevaluación y coevaluación). Ambos asumen el mismo rol en la evaluación. Analizan el proceso como un todo y no solamente como la obtención de resultados. También reflexionan sobre su conocimiento y el progreso en la Práctica de Campo, y al final se plantean sugerencias para una futura actividad. 
"(...) La evaluación debe verse como un proceso de concertación, donde maestros y estudiantes reconocen sus intereses y desarrollos particulares, para lo aval se pretende tener espacios de debate $y$ confrontación de argumentos en torno a situaciones problema, a los hallazgos realizados y el avance de los mismos (...)"

A diferencia de los resultados de las categorías de Enfoque, Finalidades y Momento de Realización, la categoría de Rol en la Evaluación da cuenta de otra perspectiva, en la cual el docente va más allá de proponer que la evaluación esté basada específicamente en preguntas puntuales y por el contrario es el docente y el estudiante los que asumen el mismo rol en la evaluación, analizando desde una perspectiva holística los resultados del Trabajo Práctico y lo mas importante es que reflexionan sobre su conocimiento y el progreso en su aprendizaje (Puentes, 2008).

Contenidos a evaluar: Con relación a los contenidos a evaluar, los formadores destacan que se evalúan contenidos conceptuales como la contextualización, procedimentales como el desarrollo de auademo y diario de campo y actitudinales como el desempeño y el interés.

"(...) El proceso evaluativo tiene tres momentos, el antes el durante y después (...) el antes es la contextualización (...) el durante es con la salida y el desempeño que tiene ese equipo de trabajo en los lugares (...) y el después es el diario de campo y el cuaderno de campo (...)"

Desde la perspectiva de Coll et al (1998), explicitar los contenidos de tipo conceptual, procedimental y actitudinal permite la realización de actividades prácticas de enseñanza con unos mejores resultados. Además le permite al formador y al futuro licenciado considerar la evaluación como una actividad formativa, procesual y continua (Puentes, 2008).

\section{Relación con aspectos epistemológicos}

Relación con el estatus epistemológico de la Biología: Los formadores explicitan el trabajo acerca de aspectos epistemológicos de la Biología, considerando sus características como una Ciencia autónoma, diferente de otras Ciencias y con criterios de validación propios. Desde la perspectiva de Mayr (1998) y Jacob (1986), la Biología actualmente, es una Ciencia diversificada. Además de esto se considera como una Ciencia autónoma (Mayr, 2006).

['Al planificar la práctica de campo] (...) Se hace referencia a la construcción del Conocimiento Biológico (...) la observación, trabajo científico (...) cómo nace la Biología como Ciencia, cómo se estructura la Biología, por qué es autónoma la Biología, por qué se considera diferente otras Ciencias (...)"

Objeto de estudio de la Biología: Aunque se encuentran pocas afirmaciones con respecto a esta categońa por parte de los formadores, se evidencia que éstos consideran que en Biología no se estudia la vida sino lo vivo.

"[Haciendo referencia al objeto de estudio de la Biología] (...) es decir que no son las características de la vida sino de lo vivo (....)"

Es fundamental reconocer que la Biología es una Ciencia, con un arerpo organizado de conocimientos, que pretende explicar el mundo de lo vivo y los fenómenos que en él se presentan (Puentes, 2008). Aunque se identifica el objeto de estudio de la vida, no se observa desde qué perspectiva se estudia ese objeto, ya que como plantea Sánchez, (2007), puede hacerse de una visión analítica, en la cual la unidad de estudio está constituida por la suma de partes, y al estudio de estas partes se le da una gran relevancia, asumiendo que para conocer el todo basta con 
conocer sus partes constituyentes; o puede hacerse desde una visión sistémica, en donde se tiene en cuenta la emergencia como característica de los sistemas vivientes y en donde el estudio de las interrelaciones es fundamental, entendiendo que no todo el Conocimiento Biológico es observable, ni experimentable, sino que es producto de la construcción conceptual (Valbuena; Castro y Sienra,. 2006).

Producción del Conocimiento Disciplinar Biológico: Los profesores formadores plantean que la construcción de Conocimiento Biológico se ve influenciado por diversos aspectos como realidades, cosmovisiones e intenciones; además se tiene en cuenta la observación, el auestionamiento de los procesos biológicos, la argumentación y la interpretación.

"(...) [Objetivo] Reconocer el proceso de construcción de conocimiento de la Biología como Ciencia, desde el cuestionamiento de los procesos biológicos observados en la Práctica de Campo (...)"

Este referente corresponde a una postura en la cual se propone que la experimentación es la base del Conocimiento Biológico (Puentes, 2008). Es de resaltar que además se reconocen las narraciones históricas como forma de producción de conocimiento particular de la Biología (Caponi, 2001).

Relación con la imagen de Ciencia: En la Práctica de Campo de primer semestre se consideran elementos acerca de la Biología que dan cuenta de una imagen de Ciencia no exduyente, construida por personas y que puede ser falseada o no, entre estos el conocimiento cientúfico, el conocimiento empírico y la validación del conocimiento.

"[haciendo referencia a la imagen de ciencia trabajada en la Práctica de Campo] (...) Comparación entre las diferentes representaciones de ése conocimiento (...) para que ellos reconozcan la importancia de ése conocimiento científico, que algunos saberes se validan, otros no (...)"

Según Porlán, Rivero y Martín del pozo, (1997), este referente podría posibilitar el acercamiento a los futuros profesores de manera real a la forma de construcción de los cientíicos y sobre todo a la idea de que la Ciencia es hecha por humanos y para humanos, que no son seres extraños quienes la producen, sino que esa producción esta mediada por los intereses, deseos y necesidades de unos seres tan humanos como ellos.

Es muy importante que en la formación inicial de docentes, además de ocuparse del aprendizaje de los aspectos básicos de cada disciplina cient́fica, también se aborden los aspectos históricos y epistemológicos, de tal forma que se propicien las reflexiones epistemológicas sobre la naturaleza del conocimiento cient́fico y las implicaciones sociales de la Ciencia (Valbuena, 2007).

\section{Aspectos relacionados con la formación docente}

Esta categonía emerge de nuestra investigación con el propósito de resaltar aquellos aspectos reflexivos que se propician con las Prácticas de Campo. En dichas prácticas, desde las diferentes guías, programas y entrevistas de los formadores, se induyen en una buena proporción elementos que involucran la reflexión sobre la realidad docente y diversos contextos educativos y fundamentalmente la formación de docentes de Biología.

"(...) [Elemento de la guía anexa relacionada con la visita a la estación astronómica precolombina muisca de Monquirá] ¿Qué elementos aporta el reconocimiento de este lugar en tu formación como licenciado en Biología? (...)" 
Este referente da cuenta que desde el enfoque del profesor investigador, es importante que el futuro profesor sea capaz de cuestionar y reestructurar sus teorías implíatas y las rutinas aprendidas en su experiencia como estudiantes y que sea además de ser un profesional autónomo, crítico, investigador un profesional reflexivo sobre su propia práctica (Garća, 2006). La reflexión en y sobre la práctica de la enseñanza permite al profesor en formación analizar su conducta en clase, contrastarla con sus conocimientos y concepciones y en un proceso de retroacción, redefinir sus conocimientos, estrategias de enseñanza y ponerlas en práctica (Mellado y Gonzáles, 2000).

\section{Aspectos relacionados con el currículo del PCLB}

Esta categoría emerge con el fin de resaltar la relación de las Prácticas de Campo con los diversos aspectos del PCLB. Para este caso los formadores explicitan como característica de la Práctica de Campo, la integración de los componentes en la misma, gracias a los espacios académicos de plenaria y tutoría. También se reconoce el aporte de la salida a la resolución del Núdeo Integrador de Problema (NIP) y a trabajos transversales del semestre.

"(...) Todo se centra en responder el NIP y al final todos los específicos de los componentes se reúnen en la respuesta y eso le colabora mucho los estudiantes en los trabajos transversales aparte de la salida (...)"

En este sentido es importante resaltar que las características de la Práctica de Campo, están mediadas por los aspectos relacionados con el curríalo, para este caso el PCLB, en donde se concibe dicha actividad como una gran integración en el semestre, sus componentes y ejes transversales. De este modo es importante tener en cuenta que durante la formación inicial no basta con que los formadores, siguiendo un modelo de orientación aditiva (Gess- Newsome, 1999), enseñen a los estudiantes-futuros licenciados los diferentes saberes académicos que componen el conocimiento profesional, de una forma desarticulada, a manera de yuxtaposición, impartiendo simultáneamente muchas cursos tanto del ámbito disciplinar espećfico como del área de la pedagogía y didáctica sin alcanzar siquiera a aproximaciones entre si y mucho menos en que exista una articulación entre ellos, pretendiendo que cuando ejerzan la profesión los integren como producto de la necesidad en la práctica.

\section{Conclusiones y proyecciones}

La Práctica de Campo planificada en primer semestre de Licenciatura en Biología, responde en su mayoría a un trabajo de tipo expositivo, en donde los formadores plantean ejercicios cenados en los que se solicita a los futuros licenciados, describir, definir, diligenciar tablas y registrar datos, daro esta que después de haber abordado lo teórico. En esta PC se plantean finalidades conceptuales, procedimentales y actitudinales relacionadas tanto con la formación disciplinar Biológica, cultural y social, como con la formación docente. Además, da cuenta de un tipo de evaluación formativa en la que tanto formadores como futuros licenciados asumen el mismo rol. En esta PC se evalúan contenidos conceptuales, procedimentales y actitudinales. Los futuros licenciados no participan en el diseño de la guía y durante la práctica ejecutan lo planteado por los formadores.

Como característica fundamental de la Práctica de Campo, destacamos la integración de los diferentes espacios académicos del semestre en la misma, gracias al trabajo del equipo docente.

Resaltamos el abordaje epistemológico que propicia la Práctica de Campo, lo cual es fundamental en primer semestre ya que se constituye en el primer referente acerca de la Biología como ciencia, destacando su relevancia en tanto es uno de los pocos semestres en lo que se aborda explícitamente dicho aspecto. 
Con respecto a la formación docente, la Práctica de Campo de primer semestre se caracteriza por potenciar la reflexión del futuro profesor acerca de su identidad docente, del sujeto maestro y del papel del docente en la escuela. Esto es importante considerando que las Prácticas de Campo son fundamentales en la formación de profesores de Biología, no sólo en tanto contribuyen a la construcción del Conocimiento Biológico, sino también a la construcción del Conocimiento Didáctico del Contenido Biológico y la reflexión de la profesión como docentes. Esto es fundamental ya que no basta con que el docente adquiera competencias para aplicar los referentes teóricos sino requiere, además, poseer un conocimiento tal que lo capacite para tomar decisiones en contextos educativos particulares, por lo que en la formación inicial del profesorado es importante que se propicien espacios para que los futuros docentes vivencien los ambientes escolares, y reflexionen sobre las idiosincrasias particulares de los profesores en ejercicio y su incidencia en el ejercicio profesional.

Teniendo en cuenta lo anterior, es importante que en la formación de maestros se trabaje porque éstos se reconozcan como sujetos de conocimientos propios de su profesión. La formación del profesorado debe partir de un enfoque reflexivo, en el que se analicen las prácticas profesionales, las tareas y los conocimientos de los maestros.

A manera de proyección, dado que esta investigación tuvo en cuenta exdusivamente la planeación de dichas actividades, es fundamental que se continúe realizando este tipo de investigaciones ahora con respecto a la estrategia formativa aplicada, desde los desarrollos en los futuros docentes.

\section{Bibliografía}

Alarcón, Y y Piñeros, I (1989). Las salidas de campo como un reaurso pedagógico. Modelo de una salida. Tesis para optar al título de Licenciado en Biología y Química. Universidad de la Salle. Bogotá.

Caponi, G (2001). Biología funcional vs Biología evolutiva. Episteme Porto Alegre 12. pp 23-46.

Coll, C; Pozo, I; Sarabia, B; Valls, E (1992). Los contenidos en la reforma. Enseñanza $y$ Aprendizaje de conceptos, procedimientos y actitudes. Madrid: Santillana.

García, E (2006). La integración de la teoría con la práctica en la formación inicial del profesorado. En Alambique. Didáctica de las Ciencias Experimentales. 47. pp 65-73.

Del Carmen, L y Pedrinaci E (1997). El uso del entomo y el trabajo de campo. En DEL CARMEN (Coord) La enseñanza y el aprendizaje de las Ciencias de la naturaleza en la educación secundaria. (pp 133-154). Barcelona: Editorial Horsori.

Geli, A (1995). La Evaluación de los Trabajos Prácticos. En: Alambique. Didáctica de las Ciencias Experimentales. 4 (2), 25-32.

Gess-Neusome, J. (1999). Pedagogical Content Knouledge: An introduction and orientation. In: Gess-Newsome, J. And Lederman, N. (Eds.). Examining Pedagogical Content Knowledge. The Construct and its Implications for Science Education. Dordredht, Boston, London: Kuwer Academic Publishers.

Gil, D. Y Valdés, P (1996). La orientación de las Prácticas de laboratorio como investigación: Un ejemplo ilustrativo. En: Enseñanza de las Ciencias. 14 (2), 155-163.

Grossman, P. (1990) the making of a teacher knouledge and teacher education, New York: Teachers college, Columbia University.

Jacob, F (1986). La Lógica de lo Viviente. Una historia de las Ciencias. Barcelona: Busquets.

Mayr, E (1998). Así es la Biología. Madrid: Editorial Debate.

Mayr, E. (2006). Por qué es única la Biología: Consideraciones sobre la Autonomía de una Disciplina Científica. Buenos Aires: Katz 
Mellado, V y Gonzáles, T (2000). La formación inicial del profesorado de ciencias. En PERALES J y Cañal P (Coord) Didáctica de las Ciencias Experimentales. (pp 535-555) Editorial Marfil Alcoy: España.

Moreno, N; Rodriguez, A; Torres, J; Mendoza, N; Latorre, L (2006). Tras las huellas del saber pedagógico. Bogotá D.C. Universidad Pedagógica Nacional.

Porlán, R; Rivero, A y Martín, R (1997). Conocimiento profesional y epistemología de los profesores I: Teońa, métodos e instrumentos. En: Enseñanza de las Ciencias. 15 (2) 155-171.

Puentes, M (2008). Propuesta de un sistema de categorías para el estudio del Trabajo Práctico en la enseñanza de la Biología. Trabajo de grado para optar al título de Especialista en Enseñanza de la Biología. Universidad Pedagógica Nacional: Bogotá D.C.

Rodrigo, M et al (1999) Concepciones sobre el Trabajo Práctico de campo (TPc): una aproximación al pensamiento de los futuros profesores. En Revista Complutense de Madrid. 10 (2), 261-285.

Sánchez, P (2007). Formulación de proposiciones para el estudio de las concepciones sobre el Conocimiento Biológico en el marco del conocimiento profesional del profesor de Biología. Universidad Pedagógica Nacional, Facultad de Ciencia y Tecnología. Departamento de Biología. Bogotá.

Tardif, M. (2004). Los saberes del docente y su desarrollo profesional. Traducción de Pablo Manzano. Madrid: Narcea.

Valbuena, E. Castro, A. Sierra, C. (2006) El conocimiento biológico desde la perspectiva del conocimiento profesional del profesor de Biología. En Memorias del $2^{\circ}$ Congreso Internacional de Enseñanza de la Biología. Neuquén, Argentina.

Valbuena, E. (2007). El Conocimiento Didáctico del Contenido Biológico. Estudio de las concepciones disciplinares y didácticas de futuros docentes de la Universidad Pedagógica Nacional (Colombia). Tesis para optar al título de Doctor en Didáctica de las Ciencias Experimentales. Universidad Complutense de Madrid. 